\section{Uso inapropiado de medicamentos en adultos mayores: resultados de la Encuesta Nacional de Salud 2010}

\author{
ALVARO PASSI ${ }^{\mathrm{a}}$, PAULA MARGOZZINI ${ }^{1}$, EDUARDO VALENZUELA ${ }^{2}$, \\ TRINIDAD HOYL ${ }^{2}$, PEDRO PAULO MARÍN ${ }^{2}$, \\ MARCELA CARRASCO ${ }^{2}$, RICARDO OLEA ${ }^{\mathrm{b}}$, HOMERO GAC $^{2}$
}

\section{Inappropriate medication use among Chilean older people}

Background: Inappropriate medication use in older people is an important source of adverse events and complications. Aim: To determine the frequency of inappropriate medication use in the general population. Material and Methods: As part of the 2010 Chilean National Health Survey, 1,048 persons aged 65 years or more were interviewed about medication use. The information obtained was analyzed using standardized Beer's criteria, adapted for the Chilean population, to define inappropriate medication use. A logistic regression model was performed to define risk factors for inappropriate medication use. Results: Ten percent of subjects had a high risk of inappropriate medication use. The most common medications used were chlorphenamine, amitriptyline, diazepam, chlorpromazine, chlordiazepoxide and piroxicam. The risk factors detected were female gender, polypharmacy and a bad health self-perception. Conclusions: Inappropriate medication use is common among Chilean older people and should be discouraged.

(Rev Med Chile 2016; 144: 417-425)

Key words: Aged; Drug-Related Side Effects and Adverse Reactions; Medication Errors.

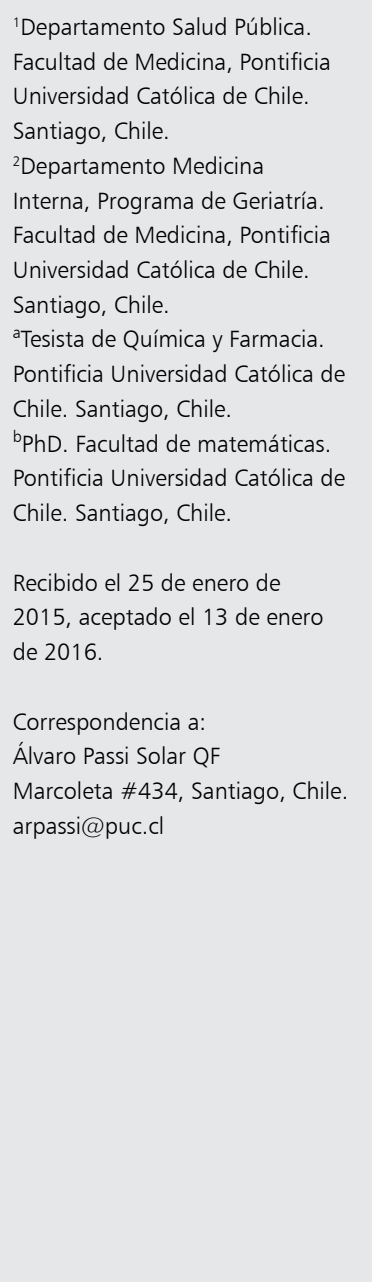
Universidad Católica de Chile. Santiago, Chile.

${ }^{2}$ Departamento Medicina Interna, Programa de Geriatría. Facultad de Medicina, Pontificia Universidad Católica de Chile.

Santiago, Chile.

aTesista de Química y Farmacia. Pontificia Universidad Católica de Chile. Santiago, Chile.

${ }^{b} \mathrm{PhD}$. Facultad de matemáticas. Pontificia Universidad Católica de

Recibido el 25 de enero de 2015, aceptado el 13 de enero Marcoleta \#434, Santiago, Chile. arpassi@puc.cl
E 1 uso inapropiado de medicamentos (UI), utilizando la definición de Beers (1991), es el uso de medicamentos que introducen un riesgo significativo de algún efecto adverso relacionado a medicamentos, cuando existe evidencia de una terapia alternativa de igual o mayor efectividad y con menor riesgo ${ }^{1}$. El UI es un problema de salud que aumenta el riesgo de reacciones adversas asociadas a medicamentos (RAM) $)^{2}$ y los problemas relacionados con ellos, como mayores costos de salud ${ }^{3}$ y empeoramiento de la calidad de vida ${ }^{2}$.

El criterio de Beers ha sido la herramienta de detección de UI más usada a nivel global, creada en 1991 y su última actualización se desarrolló en el año $2012^{1,4,5}$. A nivel mundial existen pocos estudios de población general que analicen el UI y sus resultados son difíciles de extrapolar a la población chilena, dada las diferencias en el catálogo de medicamentos disponibles.

De acuerdo a la ENS $2003^{6} 11,3 \%$ de los adultos mayores (AM) presenta este problema en Chile $y$, por lo tanto, su importancia deriva tanto de las consecuencias en salud a nivel individual y poblacional, como del impacto económico sobre el sistema de salud del país. En Chile no hay estudios que indiquen cuál es la carga económica que este problema representa, sin embargo, se reconoce que la prevención y el reconocimiento de los problemas relacionados con medicamentos en AM y en otros grupos vulnerables, es uno de los principales asuntos de seguridad y calidad de la salud por resolver? ${ }^{7}$. Es probable que el plan de 
Garantías Explícitas en Salud (GES) que comenzó en el año 2005, con su fomento a la adhesión a los protocolos de tratamientos de las patologías cubiertas, haya afectado en la prevalencia y en el perfil de UI observado en el año 2003.

El presente estudio surge de la necesidad de actualizar y caracterizar el UI de medicamentos en el AM de la población general chilena, analizando para ello los datos obtenidos por la ENS 2010.

\section{Material y Método}

El estudio se realizó en el contexto de las mediciones de la ENS $2010^{8}$, la cual es un estudio de prevalencia nacional que contempla la evaluación de 43 temas de salud prioritarios en el adulto, en una muestra aleatoria representativa de la población general chilena mayor de 14 años. La ENS 2010 recogió adicionalmente un inventario de medicamentos en uso por cada participante. Para el presente análisis se incluyó a los 1.048 individuos de 65 o más años (AM), que participaron en la ENS 2010.

El inventario de medicamentos se realizó en el hogar del entrevistado en presencia de una enfermera entrenada, la que les solicitó que mostraran los medicamentos que estaban utilizando actualmente.

Se evaluó el estado cognitivo de los AM participantes a través del test minimental modificado y el test Pfeffer.

Personal especialista en farmacología aplicó la clasificación ATC-WHO ${ }^{9}$ a los principios activos contenidos en los medicamentos registrados por el inventario.

El instrumento estándar de detección de UI creado por Beers debió ser adaptado a la realidad Chilena, ya que los principios activos (PA) clasificados como inapropiados por Beers provienen del análisis del mercado farmacéutico norteamericano. El proyecto de adaptación del criterio de Beers fue realizado por parte del programa de geriatría de la Pontificia Universidad Católica de Chile (PUC) durante el año 2006, a partir del criterio de Beers del año $2002^{4}$. El listado creado por Beers fue ampliado a los fármacos comercializados en Chile. Para adaptar el instrumento se encuestó por vía electrónica a expertos farmacólogos y geriatras chilenos, recogiendo opiniones que permitieran actualizar y adaptar a la realidad chilena dichos criterios a través del método Delphi ${ }^{10}$.
Los principios activos usados por los AM fueron clasificados, considerando el consenso de expertos en geriatría de hospital clínico PUC, en niveles de UI adaptados a Chile el año 2006 y actualizados el año 2010, aludiendo a la gravedad de las consecuencias de la indicación en adultos mayores, siguiendo las recomendaciones de Beers: 1) no se debe usar; 2) tiene excepcionales indicaciones; 3 ) se puede usar sólo en cierto grupo de adultos mayores en mejor estado funcional y 4) se puede usar sin problemas en el adulto mayor (Tablas 1, 2 y 3 ).

La clasificación que se realizó es independiente de los diagnósticos o condiciones de base del AM, ya que la ENS 2010 no incluye todos los diagnósticos necesarios para este fin.

El análisis estadístico se realizó con el programa SPSS statistics ${ }^{\circledR} \mathrm{v} .18$, en el módulo de muestras complejas, obteniendo resultados en prevalencias expandidas en virtud del muestreo complejo y ajustadas a la composición demográfica nacional, según proyecciones censales del año 2002 para enero del año 2010.

Se determinó la prevalencia e intervalos de confianza a 95\% (IC 95\%) de UI según gravedad (Niveles 1, 2, 3 y 4) y sexo.

Para determinar los factores de riesgo asociados al uso de algún PA del nivel 1 se realizó un modelo de regresión logística utilizando como descriptores las variables independientes sexo, nivel educacional (NEDU), zona urbano-rural, polifarmacia (uso de 5 o más principios activos), presencia de médico de cabecera y estado de salud. Se calcularon odds ratios (OR), con sus respectivos intervalos de confianza a 95\% (IC 95\%), para cada una de estas variables. El estado de salud corresponde a la salud autopercibida en categorías de "muy buena", "buena", "más o menos",

\section{Tabla 1. Características de la población de 65 años y más en Chile, Encuesta Nacional de Salud chilena 2009-10}

\begin{tabular}{|cl|}
\hline Gravedad & Definición \\
\hline Nivel 1 & No se debe usar \\
Nivel 2 & Tiene excepcionales indicaciones de uso \\
Nivel 3 & $\begin{array}{l}\text { Se pude usar sólo en cierto grupo de AM } \\
\text { en mejor estado funcional }\end{array}$ \\
Nivel 4 & Se puede usar sin problemas en el AM \\
\hline
\end{tabular}


Tabla 2. Listado de principios activos usados por la población adulta de 65 años y más según uso inapropiado Nivel 1, 2, y 3. Encuesta Nacional de Salud chilena 2010

\begin{tabular}{|c|c|c|}
\hline \multicolumn{3}{|l|}{ Nivel 1} \\
\hline Amitriptilina & Clorpropamida & Metildopa \\
\hline Bromuro de pipenzolato & Dexclorfeniramina & Metoclopramida \\
\hline Cisaprida & Diazepam & Pentaeritrilo tetranitrato \\
\hline Clordiazepóxido & Efedrina clorhidrato & Piroxicam \\
\hline Clorfenamina maleato & Fenilbutazona & Pseudoefedrina \\
\hline Clormezanona & Imipramina & Tioridazina \\
\hline \multicolumn{3}{|l|}{ Clorpromazina } \\
\hline \multicolumn{3}{|l|}{ Nivel 2} \\
\hline Acetazolamida & Clonazepam & Lorazepam \\
\hline Alprazolam & Clopidogrel & Mebeverina \\
\hline Amiodarona & Cloramfenicol & Metamizol sódico (dipirona) \\
\hline Atropina & Codeína fosfato & Metilprednisolona, oral \\
\hline Baclofeno & Diclofenaco sódico & Metotrexato \\
\hline Beclometasona & Estradiol & Modafinilo \\
\hline Brinzolamida & Estrógenos conjugados & Nimodipino \\
\hline Bromazepam & Fenitoina & Papaverina \\
\hline Bromuro de pinaverio & Fenobarbital & Pargeverina \\
\hline Bromuro de tiotropio & Fenolftaleina & Pramipexol \\
\hline Calcio cloruro & Flunarizina & Rosiglitazona \\
\hline Carbamazepina & Glipizida & Sen \\
\hline Ciclobenzaprina & Griseofulvina & Sodio selenato \\
\hline Cinarizina & Haloperidol & Sotalol \\
\hline Clenbuterol & Indometacina & Tolbutamida \\
\hline Clobazam & Litio carbonato & \\
\hline \multicolumn{3}{|l|}{ Nivel 3} \\
\hline Ácido nicotínico & Fluconazol, oral & Naproxeno sódico \\
\hline Betacaroteno & Fluoxetina & Nifedipino \\
\hline Betametasona & Flutamida & Nitrofurantoina \\
\hline Bezafibrato & Fluticasona & Oxibutinina \\
\hline Bromhexina & Galantamina & Prednisona \\
\hline Bromocriptina & Ginkgo billoba & Quetiapina \\
\hline Buspirona & Hidroxicloroquina & Risperidona \\
\hline Cafeina & Indapamida & Rutosido \\
\hline Calcio glicerofosfato & Isosorbida dinitrato & Saccharomyces boulardii \\
\hline Celecoxib & Ketoprofeno & Salmeterol \\
\hline Cilostazol & Ketorolaco & Sitagliptina \\
\hline Desloratadina & Levocetirizina & Terbinafina, oral \\
\hline Diclofenaco & Loratadina & Tibolona \\
\hline Digoxina & Meloxicam & Tolterodina \\
\hline Duloxetina & Mianserina & Tramadol \\
\hline Ergotamina & Mirtazapina & Zolpidem \\
\hline Fenoterol & & \\
\hline
\end{tabular}


Tabla 3. Listado de principios activos uso inapropiado Nivel 4 usados por la población adulta de 65 años y más. Encuesta Nacional de Salud chilena 2010

\begin{tabular}{|c|c|c|c|}
\hline \multicolumn{4}{|l|}{ Nivel 4} \\
\hline Acenocumarol & Cianocobalamina & Lactobacillus acidophilus & Ranitidina \\
\hline Ácido acetilsalicílico & Ciprofloxacino clorhidrato & Lactulosa & Retinol \\
\hline Ácido alendrónico & Citalopram bromhidrato & Lamivudina & Riboflavina \\
\hline Ácido ascórbico & Cloxacilina & Lansoprazol & Rivastigmina \\
\hline Ácido fólico & Colchicina & Levetiracetam & Rosuvastatina \\
\hline Ácido ibandrónico & Colecalciferol & Levodopa & Salbutamol \\
\hline Ácido valproico & Condroitin sulfato & Levofloxacino & Salbutamol \\
\hline Aliskireno & Deflazacort, oral & Levotiroxina de sodio & Selenio \\
\hline Alopurinol & Diltiazem & Loperamida & Sertralina \\
\hline Aluminio hidróxido & Diosmina & Losartan & Simvastatina \\
\hline Ambroxol & Domperidona & Lovastatina & Sodio fosfato \\
\hline Amilorida & Dorzolamina & Magaldrato & Sucralfato \\
\hline Aminofilina & Doxazosina & Magnesio & Sulfametoxazol \\
\hline Amlodipino & Doxiciclina & Magnesio carbonato & Sulfasalazina \\
\hline Amonio cloruro & Doxilamina & Metformina & Sulfato cúprico \\
\hline Amoxicilina & Enalapril & Mometasona & Tamoxifeno \\
\hline Atenolol & Ergocalciferol & Mononitrato de isosorbida & Tamsulosina \\
\hline Atorvastatina & Escitalopram & Multivitamínicos & Telmisartán \\
\hline Betacaroteno & Esomeprazol & Nebivolol & Teofilina anhidra \\
\hline Betametasona dipropionato & Espironolactona & Nevirapina & Tiamina \\
\hline Betaxolol & Ezetimiba & Nicotinamida & Timolol \\
\hline Biotina & Famotidina & Nicotinato de inositol & Tocofersolán \\
\hline Bisoprolol & Fitomenadiona & Nitrendipino & Travoprost \\
\hline Bromuro de clidinio & Fluconazol & Noscapina & Trazodona \\
\hline Bromuro de ipatropio & Fluoruro sodico & Omeprazol & Triamcinolona acetonida \\
\hline Bromuro de ipatropio & Fluticasona furoato & Pantoprazol & Triamtereno \\
\hline Budesonida & Furazolidona & Pantotenato de calcio & Triglicéridos omega 3 \\
\hline Budesonida, oral & Furosemida & Paracetamol & Trihexifenidilo \\
\hline Calcio carbonato & Gemfibrozilo & Paroxetina & Trimebutina \\
\hline Calcio fosfato dibásico & Glibenclamida & Pentoxifilina & Trimetoprima \\
\hline Calcio gluconato & Glucosamina & Perindoprilato & Vacuna combinada DTP \\
\hline Calcio, sal no identificada & Hidralazina & Picosulfato de sodio & adsorbida antidifteria, \\
\hline Calcitriol & Hidroclorotiazida & Piridoxina & tetanos y tos \\
\hline Candesartan & Hidroxocobalamina clorhidrato & Piritinol & Valsartán \\
\hline Capsaicina & Ibuprofeno & Potasio citrato monohidrato & Valsartán \\
\hline Captopril & Insulina humana & Potasio gluconato & Valsartán \\
\hline Carbocisteina & (ADN recombinante) & Procaína & Zidovudina \\
\hline Carvedilol & loduro de potasio & Propranolol & Zinc óxido \\
\hline Cetirizina & Ketoconazol, oral & Ramipril & Zopiclona \\
\hline
\end{tabular}


"mala" y "muy mala", se analizó como variable numérica discreta (con valores de 1 a 5), y el valor calculado en la regresión logística corresponde al OR de empeorar la salud en una categoría (pasar de "muy buena" a "buena" o de "buena" a "más o menos", sucesivamente). Se caracterizó la fuerza de determinación del modelo construido a través del pseudo $\mathrm{R}^{2}$, calculado a partir del método de Nagel kerke ${ }^{11}$.

Al margen de las tablas de resultados se muestra la cantidad de individuos de la muestra no ponderada $(n)$, y las prevalencias o promedios de la muestra expandida.

\section{Resultados}

La muestra expandida de los 1.048 adultos mayores de la ENS 2010 representó a 1.589.261 de AM, correspondientes a $12 \%$ de la población de 15 años y más, lo cual concuerda con las proyecciones censales del Instituto Nacional de Estadísticas (INE) para enero de 2010. El rango de edad de los participantes está entre 65 y 100 años, siendo la media de 73,5 años, $42,3 \%$ son hombres, la mayor parte de esta población tiene un nivel educacional bajo, son de la zona urbana y viven en la Región Metropolitana (RM) (Tabla 4).

El 9,7\% (IC95\%: 7,1\%-13,1\%) de los AM consume al menos un PA el cual está clasificado como UI nivel 1 (no se debe usar) y las mujeres presentaron una prevalencia significativamente mayor a la obtenida por los hombres. El 25,5\% usa al menos un PA del nivel 2 (tiene excepcionales indicaciones) y $31,4 \%$ usa al menos un PA del nivel 3 (se puede usar sólo en AM con mejor estado funcional). No existen diferencias signifi- cativas según sexo para estos últimos dos niveles de UI (Tabla 5).

$\mathrm{Al}$ usar en la regresión logística multivariada para el UI Nivel 1 (no se debe usar) las variables edad, sexo, NEDU, zona, polifarmacia, autopercepción del estado de salud y presencia de médico de cabecera se obtuvo un pseudo $\mathrm{R}^{2}$ de 0,208 . Las variables que resultaron ser independientemente asociadas al UI de Nivel 1 (Tabla 6) fueron el sexo femenino, con un OR de 4,6 (IC95\%: 2,1$10,1)$, presencia de polifarmacia, con un OR de 2,8 (IC95\%: 1,3-6,1), y la autopercepción del estado de salud, con un OR de 1,6 al comparar los estados de salud contiguos (salud "buena" vs "muy buena", "más o menos" vs "buena", "mala" vs "más o menos", y "muy mala" vs "mala"). El nivel educacional, la zona de vivienda urbano-ru-

Tabla 4. Características de la población de 65 años y más en Chile,

Encuesta Nacional de Salud chilena 2010

\begin{tabular}{|c|c|c|}
\hline Característica & $\mathbf{n}$ & $\begin{array}{l}\text { Estimación } \\
\% \quad \text { (IC95) }\end{array}$ \\
\hline Edad (x) & 1.048 & $73,5 \quad(72,8-74,2)$ \\
\hline $\begin{array}{l}\text { Sexo \% } \\
\text { Hombres } \\
\text { Mujeres }\end{array}$ & $\begin{array}{l}406 \\
642\end{array}$ & $\begin{array}{ll}42,3 & (37,0-47,8) \\
57,7 & (52,2-63,0)\end{array}$ \\
\hline $\begin{array}{l}\text { NEDU\% } \\
\text { Bajo (Menor a } 8 \text { años) } \\
\text { Medio (8 a } 12 \text { años) } \\
\text { Alto (Más de } 12 \text { años) }\end{array}$ & $\begin{array}{r}688 \\
268 \\
58\end{array}$ & $\begin{array}{rr}57,4 & (51,8-62,8) \\
29,9 & (25,2-35,2) \\
12,7 & (8,7-18,1)\end{array}$ \\
\hline $\begin{array}{l}\text { Zona\% } \\
\text { Urbano } \\
\text { Rural }\end{array}$ & $\begin{array}{l}865 \\
183\end{array}$ & $\begin{array}{ll}81,4 & (76,4-85,5) \\
18,6 & (14,5-23,6)\end{array}$ \\
\hline
\end{tabular}

Tabla 5. Prevalencia de uso inapropiado de medicamentos en población de 65 años y más según gravedad (nivel) y sexo, Encuesta Nacional de Salud chilena 2010

\begin{tabular}{|c|c|c|c|c|c|c|}
\hline Gravedad & $\mathbf{n}$ & $\begin{array}{l}\text { Hombres } \\
\text { Prevalencia } \\
\% \quad(I C 95 \%)\end{array}$ & $\mathbf{n}$ & $\begin{array}{l}\text { Mujeres } \\
\text { Prevalencia } \\
\% \quad(I C 95 \%)\end{array}$ & $\mathbf{n}$ & $\begin{array}{l}\text { mbos sexos } \\
\text { Prevalencia } \\
\% \quad(I C 95 \%)\end{array}$ \\
\hline Nivel 1 & 367 & $4,1 \quad(2,1-7,9)$ & 577 & $14,0 \quad(9,9-19,3)$ & 944 & $9,7 \quad(7,1-13,1)$ \\
\hline Nivel 2 & 367 & $24,1 \quad(15,5-35,4)$ & 577 & $26,7 \quad(21,3-32,8)$ & 944 & $25,5 \quad(20,5-31,3)$ \\
\hline Nivel 3 & 367 & $29,5 \quad(20,5-40,4)$ & 577 & $32,9(26,7-39,8)$ & 944 & $31,4 \quad(26,1-37,4)$ \\
\hline Nivel 4 & 367 & $61,3 \quad(51,8-70,1)$ & 577 & $68,3 \quad(61,3-74,5)$ & 944 & $65,3 \quad(59,7-70,5)$ \\
\hline
\end{tabular}


Tabla 6. Variables explicatorias del UI Nivel 1 (no se debe usar) en población de 65 años y más. Encuesta Nacional de Salud chilena 2010

\begin{tabular}{|c|c|c|}
\hline Variables & & Odds Ratio (IC95\%) \\
\hline Sexo & $\begin{array}{l}\text { Hombres } \\
\text { Mujeres }\end{array}$ & $\begin{array}{l}1,0^{\mathrm{a}} \\
4,6(2,1-10,1)\end{array}$ \\
\hline Nivel educacional & $\begin{array}{l}\text { Bajo } \\
\text { Medio } \\
\text { Alto }\end{array}$ & $\begin{array}{l}3,5(0,7-16,9) \\
1,4 \quad(0,2-8,5) \\
1,0^{\text {a }}\end{array}$ \\
\hline Zona & $\begin{array}{l}\text { Urbano } \\
\text { Rural }\end{array}$ & $\begin{array}{l}1,0^{\mathrm{a}} \\
0,5 \quad(0,2-1,3)\end{array}$ \\
\hline Polifarmacia & $\begin{array}{l}\text { Sí } \\
\text { No }\end{array}$ & $\begin{array}{l}2,8(1,3-6,1) \\
1,0^{\text {a }}\end{array}$ \\
\hline ¿Cómo calificaría hoy su estado de salud? & Al empeorar la percepción del estado de salud & $1,6(1,1-2,6)$ \\
\hline Edad & $\begin{array}{l}65-84 \text { años } \\
85 \text { años o más }\end{array}$ & $\begin{array}{l}1,0^{\mathrm{a}} \\
0,3 \quad(0,1-1,6)\end{array}$ \\
\hline Presencia de médico de cabecera & $\begin{array}{l}\text { Sí } \\
\text { No }\end{array}$ & $\begin{array}{l}1,2 \quad(0,5-2,9) \\
1,0^{a}\end{array}$ \\
\hline
\end{tabular}

aReferencia; ' $\mathrm{OR}$ ajustado por Sexo, Nivel educacional, Zona urbano-rural, polifarmacia, autopercepción del estado de salud, edad y presencia de médico de cabecera.

ral, la edad y la presencia de médico de cabecera no resultaron ser predictores estadísticamente significativos del UI nivel 1.

De los PA clasificados como UI nivel 1, la clorfenamina es la que tiene la mayor prevalencia de uso $(2,1 \%)$, seguida por amitriptilina $(1,9 \%)$, diazepam (1,2\%)(Tabla 7$)$.

Tabla 7. Prevalencia de uso de drogas de tipo nivel 1 en la población adulta de 65 años y más. Encuesta Nacional de Salud chilena 2010

\begin{tabular}{|lcc|}
\hline Principio activo & $\mathbf{n}$ & $\begin{array}{c}\text { Prevalencia } \\
\text { \% (IC95\%) }\end{array}$ \\
\hline Clorfenamina & 21 & $2,1(1,1-4,0)$ \\
Amitriptilina & 14 & $1,9(0,9-4,0)$ \\
\hline Diazepam & 13 & $1,2(0,5-2,7)$ \\
Clorpromazina & 5 & $1,1(0,4-3,3)$ \\
\hline Clordiazepoxido & 8 & $1,0(0,4-2,5)$ \\
\hline Piroxicam & 11 & $0,8(0,3-2,0)$ \\
\hline Metoclopramida & 10 & $0,6(0,3-1,5)$ \\
\hline
\end{tabular}

*Como caso especial en este informe se observa el $n$ de casos de uso para cada PA del Nivel 1. El n total para este análisis fue de 944 (367 hombres y 577 mujeres).

\section{Discusión}

El presente estudio permitió determinar la magnitud del problema de UI de medicamentos de mayor gravedad (nivel 1) en los AM chilenos, encontrándose que afecta a uno de cada diez y que se distribuye diferencialmente en la población, siendo más prevalente en las mujeres, entre aquellos que presentan polifarmacia y entre los que autoperciben tener un peor estado de salud.

Las mujeres consultan con mayor frecuencia que los hombres los centros de salud y por lo tanto están más expuestas a usar algún medicamento (aumentando la probabilidad de uso algún PA inapropiado nivel 1). Esta asociación ha sido identificada por otros estudios de base poblacional $^{12-14}$.

A mayor número de medicamentos en uso, mayor es la probabilidad de UI. Concordante con esto, la polifarmacia es un factor de riesgo para el UI ya identificado por otros estudios, siendo catalogado por algunos como el mayor determinante ${ }^{15,16}$.

Se puede esperar mayor uso de medicamentos entre los individuos que se acercan a una autopercepción de salud "muy mala", aumentando la probabilidad de presentar alguno que sea in- 
apropiado. También puede ocurrir que el UI Nivel 1 empeore el estado de salud. Un estudio ${ }^{15}$ ha reportado la relación que existe entre un peor estado de salud y el riesgo de presentar UI y otro ${ }^{17}$, determinó que los individuos que consumen medicamentos inapropiados empeoran su percepción del estado de salud con el tiempo.

Respecto a los principios activos del UI nivel 1, la clorfenamina es el más prevalente, corresponde aun antihistamínico de primera generación, que puede provocar somnolencia y efectos anticolinérgicos, y no debiera usarse dada la existencia de antihistamínicos de nuevas generaciones, más seguros para los AM. La amitriptilina es el segundo UI nivel 1 más prevalente, es un antipsicótico de primera generación, con efecto anticolinérgicos acentuados, y no debiera usarse dado que existen antipsicóticos de nuevas generaciones. El clonazepam, tercer UI nivel 1 más prevalente, tiene un largo efecto somnífero y no debiera usarse dada la existencia de benzodiacepinas de acción corta.

Este es el segundo estudio realizado en Chile que entrega información acerca del UI de medicamentos en la población general de adultos mayores de 64 años, en una muestra representativa nacional. El primer estudio se realizó a partir de la base de datos obtenidos en la ENS 2003, donde se encontró que $11,7 \%(9,0-15,1)$ de los AM presentaron UI de nivel $1^{18}$. Según el presente estudio, 9,7\% (6,6-12,5) de los AM presentó UI de nivel 1. Estas prevalencias no se pueden comparar con facilidad, ya que el listado y la cantidad de PA en el nivel 1 son diferentes, debido a que se agregaron nuevos PA y se cambiaron de nivel de gravedad otros PA, de acuerdo a la nueva información disponible. Al comparar las prevalencias puntuales de los PA más consumidos del nivel 1 de la ENS $2003^{18}$ y de la ENS 2010, se observa que la mayoría disminuyó. A pesar que no es posible indicar si la diferencia es significativa, ya que los valores del año 2003 fueron publicados sin intervalos de confianza, existe una tendencia general a la disminución de los PA más inapropiados para los AM, sugiriendo una mejora en la seguridad de uso de medicamentos en el AM.

Si sumamos los niveles de gravedad 1 y 2 las prevalencias de UI en Chile resultan llamativamente altas (mayores a 30\%) al compararse con estudios internacionales que utilizan el criterio de Beers, los cuales reportan prevalencias que varían de $15,1 \%{ }^{12}$ a $20,0 \%{ }^{19}$. Sin embargo, gran parte de esta diferencia está explicada porque los estudios no son exactamente comparables. De hecho, los criterios usados en Chile corresponden a un listado que incluye una mayor cantidad de PA que los usados por esos estudios.

Una de las limitaciones del estudio, que deriva del diseño transversal de la ENS, es que los datos no aportan la mejor evidencia para atribuir causalidad, y no podemos saber, por ejemplo, si la polifarmacia o una peor autopercepción del estado de salud aumentan el UI, o si es al revés. No obstante, el sexo femenino fue asociado como un factor de riesgo para el UI, y no es afectado por esta limitación, dado que el sexo precede de forma irrefutable al UI.

Dado que la ENS no indica la razón de uso del medicamento, se limita el análisis UI nivel 2 y 3 , pero no afecta al análisis del uso inapropiados más graves (nivel 1), dado que no se deben usar en ningún contexto.

La validez de la información recogida sobre medicamentos en la población de AM ha sido algunas veces cuestionada. Sin embargo, hay que destacar que en este estudio sólo $6,2 \%$ no pudo mostrar los productos físicamente a la enfermera, no recordaban ninguno o los mencionó sólo verbalmente ( $\sin$ mostrar la caja). Esta cifra es menor que lo observado en el resto más joven de la ENS $2010(12,9 \%)$, por lo que se estima que la validez de la información recogida es al menos similar a la que se obtiene en estudios a otras edades.

Una hipótesis que podría explicar, en parte, la alta prevalencia de UI nivel 1 en Chile se relaciona con la disponibilidad de los PA del nivel 1 (y de sus alternativas más seguras) en el arsenal de medicamentos de los servicios públicos, considerando que la mayoría de los AM son beneficiarios de estos servicios. Para poder verificar esta última hipótesis es necesario realizar un estudio de los arsenales terapéuticos y la real disponibilidad de éstos en el sistema público. Además de esta necesidad de información, surgen otras interrogantes relevantes para la salud pública, analizables por la ENS, tales como ¿Cómo se distribuye el UI al analizar el lugar de adquisición del medicamento? ¿La automedicación es un factor de riesgo para el UI? ¿los medicamento usados de forma esporádica son los que aportan más al UI? Surgen también interrogantes que requieren otras fuentes de información, tales como: ¿Afectará la implementación de un sistema 
educativo dirigido a los prescriptores sobre los las prevalencias de UI? ¿Cuál es la carga económica que tiene el UI para el sistema de salud?

Como el presente estudio se basa en la información de la población general en los hogares, es capaz de revelar lo que las personas actualmente ingieren, independiente de si fue automedicado o de la duración de su uso, y, por otra parte, como es de una muestra representativa de la población general de AM chilenos, permite evaluar la magnitud real del consumo y problemas asociados al consumo, evitando los sesgos propios de otras fuentes de información. Como el levantamiento de datos de la ENS 2010 duró 12 meses, no existe el riesgo de estacionalidad del estudio. Este estudio es un aporte para evaluar el impacto de futuras política públicas de medicamentos en Chile, identificando algunos medicamentos cuyo uso se debe desincentivar por existir evidencia de mayor riesgo que otras drogas disponibles y así disminuir sus consecuencias, tanto para la calidad de vida de los pacientes, como para la carga que este problema implica para el Sistema de Salud.

Agradecimientos: La ENS 2010 fue financiada por MINSAL. Se agradece al CITUC y al grupo de expertos en Farmacología y Geriatría que ayudaron a adaptar los criterios de Beers para Chile (U. de Chile, Sociedad Chilena de farmacología clínica, programa de Geriatría UC).

\section{Referencias}

1. Beers MH, Ouslander JG, Rollingher I, Reuben DB, Brooks J, Beck JC. Explicit criteria for determining inappropriate medication use in nursing home residents. UCLA Division of Geriatric Medicine. Arch Intern Med 1991; 151 (9): 1825-32.

2. Jano E, Aparasu RR. Healthcare outcomes associated with beers' criteria: a systematic review. Ann Pharmacother 2007; 41 (3): 438-47.

3. Fu AZ, Jiang JZ, Reeves JH, Fincham JE, Liu GG, Perri M 3rd. Potentially inappropriate medication use and healthcare expenditures in the US community-dwelling elderly. Med Care 2007; 45 (5): 472-6.

4. Fick DM, Cooper JW, Wade WE, Waller JL, Maclean JR, Beers $\mathrm{MH}$. Updating the Beers criteria for potentially inappropriate medication use in older adults: results of a US consensus panel of experts. Arch Intern Med 2003; 163 (22): 2716-24.
5. American Geriatrics Society updated Beers Criteria for potentially inappropriate medication use in older adults. J Am Geriatr Soc 2012; 60 (4): 616-31.

6. Minsal 2003. Informe final ENS 2003. Disponible en: www.encuestasalud.cl.

7. Minsal. Uso racional de medicamentos: una tarea de todos [Internet]. 2010. Available from: http://web.minsal. cl/portal/url/item/8da19e5eac7b8164e04001011e012993. pdf

8. Minsal. Departamento de epidemiología. Informe final Encuesta Nacional de Salud 2010 (ENS 2010) [Internet]. 2010 [cited 2013 Sep 11]. Available from: http:// epi.minsal.cl/estudios-y-encuestas-poblacionales/ encuestas-poblacionales/encuesta-nacional-de-salud/ resultados-ens/

9. WHOCC-ATC/DDD Index [Internet]. [cited $2012 \mathrm{Jul}$ 7]. Available from: http://www.whocc.no/atc_ddd_index/

10. Dalkey NC. The Delphi Method [Internet]. 1969 [cited 2012 Jul 7]. Available from: http://www.rand.org/pubs/ research_memoranda/RM5888.html

11. Nagelkerke NJD. A note on a general definition of the coefficient of determination. Biometrika 1991; 78 (3): 691-2.

12. Charlesworth CJ, Smit E, Lee DSH, Alramadhan F, Odden MC. Polypharmacy Among Adults Aged 65 Years and Older in the United States: 1988-2010. J Gerontol A Biol Sci Med Sci 2015; 70 (8): 989-95.

13. Morin L, Fastbom J, Laroche M-L, Johnell K. Potentially inappropriate drug use in older people: a nationwide comparison of different explicit criteria for population-based estimates. Br J Clin Pharmacol 2015; 80 (2): 315-24.

14. Narayan SW, Nishtala PS. Prevalence of potentially inappropriate medicine use in older New Zealanders: a population-level study using the updated 2012 Beers criteria. J Eval Clin Pract 2015; 21 (4): 633-41.

15. Bongue B, Naudin F, Laroche M-L, Galteau M-M, Guy $\mathrm{C}$, Guéguen R, et al. Trends of the potentially inappropriate medication consumption over 10 years in older adults in the East of France. Pharmacoepidemiol Drug Saf 2009; 18 (12): 1125-33.

16. Lapi F, Pozzi C, Mazzaglia G, Ungar A, Fumagalli $S$, Marchionni N, et al. Epidemiology of suboptimal prescribing in older, community dwellers: a two-wave, population-based survey in Dicomano, Italy. Drugs Aging 2009; 26 (12): 1029-38.

17. Fu AZ, Liu GG, Christensen DB. Inappropriate medication use and health outcomes in the elderly. J Am Geriatr Soc 2004; 52 (11): 1934-9.

18. Margozzini P. Caracterización del consumo de medica- 
Uso inapropiado de medicamentos en adultos mayores: ENS 2010 - A. Passi et al

mentos en población general Chilena: Resultados de la Encuesta Nacional de Salud ENS 2003, proyecto FONIS SA05I20052. Departamento de Salud Pública, Pontificia Universidad Católica de Chile. Facultad de Medicina. Santiago, Chile. 2007.
19. Van der Hooft CS, Jong GW't, Dieleman JP, Verhamme KMC, van der Cammen TJM, Stricker BHC, et al. Inappropriate drug prescribing in older adults: the updated 2002 Beers criteria-a population-based cohort study. $\mathrm{Br}$ J Clin Pharmacol 2005; 60 (2): 137-44. 